\title{
MONITORAMENTO DO NÍVEL DE RUÍDO DURANTE JORNADA DE TRABALHO DE COLABORADORES DO SETOR DE MANUTENÇÃO
}

\author{
C.E.L.ANDRADE ${ }^{1}$; B.A.S.MACHADO² \\ ${ }^{1}$ Centro Universitário SENAI CIMATEC; Salvador - BA \\ Mestrando em Modelagem Computacional e Tecnologia Industrial \\ celaeq@gmail.com \\ ${ }^{2}$ Centro Universitário SENAI CIMATEC; Salvador - BA; \\ brunam@fieb.org.br
}

\section{Resumo:}

A Saúde Ocupacional avança numa proposta interdisciplinar, com base na Higiene Industrial, relacionando ambiente de trabalho, corpo do trabalhador e incorpora a teoria da multicausalidade, na qual um conjunto de fatores de risco é considerado na produção da doença, avaliada através da clínica médica e de indicadores ambientais e biológicos de exposição e efeito. Fisicamente, o ruído pode ser definido como um som ou um complexo de sons indesejáveis, que causam incômodo e neuroses agudas. Afeta, física e psicologicamente, o ser humano e, dependendo dos níveis, causa lesões auditivas irreversíveis no trabalhador, podendo levar à surdez permanente. Dessa forma, o objetivo desse estudo foi verificar o nível de ruído em um ambiente de trabalho de colaboradores que exercem suas atividades no setor de manutenção. $O$ estudo foi conduzido em uma empresa localizada na cidade de Mata de São João com atuação na área de turismo. A coleta de dados ocorreu entre os dias de 22 de julho a 15 de dezembro de 2017, utilizando um áudio dosímetro e os dados amostrais do ruído foram coletados durante a jornada de trabalho de 10 colaboradores previamente selecionados conforme o Programa de Prevenção de Riscos Ambientais da empresa. Cada amostra obtida correspondeu a um tempo superior a $70 \%$ da jornada diária de trabalho de cada colaborador. Os valores das leituras de ruído foram entre 75,6 dB (para o colaborador com menor exposição) a 86,0 dB (para o colaborador com maior exposição), e a média obtida foi de $81,75 \pm 2,99 \mathrm{~dB}(\mathrm{~A})$. A partir dos resultados obtidos, identificou-se que as médias dos níveis de ruído apresentamse superiores ao nível de ação recomendado pela NR-15, que estabelece o padrão de $85 \mathrm{~dB}(\mathrm{~A})$ / 8,0 de exposição diária.

Palavras-chave: segurança do trabalho; risco; ruído; gestão.

\section{STUDY OF THE PRESENCE OF HPAS IN ATMOSPHERIC AIR SAMPLES FROM INDOORS ENVIRONMENTS - A BRIEF BIBLIOGRAPHIC PROSPECTION}

\author{
Abstract: \\ Occupational Health advances an interdisciplinary proposal, based on Industrial \\ Hygiene, relating the work environment, the body of the worker and incorporates the \\ theory of multi-causality, in which a set of risk factors is considered in the production
}


of the disease, evaluated through medical and clinical practice, environmental and biological indicators of exposure and effect. Physically, noise can be defined as a sound or a complex of unwanted sounds that cause discomfort and acute neuroses. It affects physically and psychologically the human being and, depending on the levels, causes irreversible hearing damage in the worker, which can lead to permanent deafness. Thus, the objective of this study was to verify the noise level in a work environment of employees who perform their activities in the maintenance sector. The study was conducted in a company located in the city of Mata de São João with expertise in tourism. Data collection took place between July 22 and December 15, 2017, using an audiometer and the noise sample data were collected during the workday of 10 employees previously selected according to the company's Environmental Risk Prevention Program. Each sample obtained corresponded to a time greater than $70 \%$ of the daily workday of each employee. Noise readings ranged from $75.6 \mathrm{~dB}$ (for the employee with the lowest exposure) to $86.0 \mathrm{~dB}$ (for the employee with the highest exposure), and the average obtained was $81.75 \pm 2.99 \mathrm{~dB}(\mathrm{~A})$. From the results obtained, it was found that the average noise levels are higher than the action level recommended by the NR-15, which establishes the standard of $85 \mathrm{~dB}(\mathrm{~A})$ / 8.0 daily exposure.

Keywords: workplace safety; risk; noise; management.

\section{INTRODUÇÃO}

A saúde é uma condição que abrange e inclui diversos aspectos da vida em sociedade e da própria natureza do ser humano. De acordo com a OMS (Organização Mundial da Saúde), saúde não é apenas a ausência de doença, mas também pode ser entendida como a situação de perfeito bem-estar físico, mental e social do ser humano [1]. Além disso, um importante aspecto da vida humana é o trabalho, tendo em vista que é no trabalho que muitas pessoas passam a maior parte de seu tempo desperto. A maioria das pessoas desenvolvem suas atividades profissionais em organizações produtivas e são estas organizações que podem prevenir a doença ligada ao trabalho e promover a saúde dos seus funcionários e colaboradores.

Em seu estudo, Giannini [2] afirma que o ser humano está de forma contínua recebendo informações sonoras, sejam desejáveis ou indesejáveis. Dessa forma, podem-se considerar todos os sons como ruídos, mas a sua classificação é relativamente subjetiva, destacando o fato de ser ou não desejável. Fisicamente, o ruído pode ser definido como um som ou um complexo de sons indesejáveis, que causam incômodo e neuroses agudas, afetando física e psicologicamente o ser humano e, dependendo dos níveis, causa lesões auditivas irreversíveis no indivíduo, podendo levar à surdez permanente [3]. De acordo com Ilda [4], o ruído é uma mistura complexa de diversas vibrações, medido em uma escala logarítmica em uma unidade chamada decibel $(\mathrm{dB})$.

É importante destacar que a Conferência da Terra (ECO 92), realizada no Rio de Janeiro em 1992, endossou a Agenda 21, um programa de ação mundial para a promoção do desenvolvimento sustentável, que envolve modificação de conceitos e práticas referentes ao desenvolvimento econômico e social. Neste contexto, o ruído naquela época foi considerado a terceira maior causa de poluição ambiental, atrás da 
poluição da água e do ar. Atualmente este problema ainda persiste e de acordo como o Ministério da Saúde [5], o ruído pode ser visto como o risco de agravo à saúde que atinge maior número de trabalhadores, e dessa forma, foi um dos elementos contemplados como alvo desse trabalho.

Ressalta-se ainda que desde a época taylorista, a preocupação com o bem-estar e a produtividade das pessoas que realizavam as tarefas tem sido um objetivo de organização do trabalho. Mais recentemente, esta preocupação tem se acentuado, principalmente com a introdução de normas e dispositivos legais mais rigorosos de prevenção de doenças e promoção da saúde ocupacional. Falar da organização do trabalho passou a ser falar das pessoas, das suas condições de vida no trabalho [6]. Dessa forma, o objetivo desse estudo foi verificar o nível de ruído em um ambiente de trabalho de colaboradores que exercem suas atividades no setor de manutenção em uma empresa privada da área de turismo localizada na cidade de Mata de São João no estado da Bahia.

\section{METODOLOGIA}

\subsection{Local do estudo}

O estudo foi desenvolvido em uma empresa do ramo de turismo na cidade Mata de São João (Bahia) entre os meses de julho a dezembro de 2017. A área de manutenção onde atuava colaboradores de diferentes funções foi selecionada para coleta das amostras deste estudo.

\subsection{População e amostragem}

O nível de ruído foi avaliado na área de manutenção da empresa selecionada, utilizando-se Audio Dosímetro 897 Simpson, com áudio calibrador operando em circuito de compensação "A" e circuito de resposta lenta (slow). Neste estudo, todas as medições foram realizadas próximas à orelha do trabalhador, conforme Portaria no 3.214/78 [7]. Todos os dez colaboradores incluídos no estudo tinham acesso a protetores auditivos do tipo concha. $\mathrm{O}$ uso dos protetores auditivos era exigido e fiscalizado em toda a área de manutenção, independentemente do nível de exposição ao qual o colaborador estava exposto.

Os dados amostrais do ruído foram coletados durante a jornada de trabalho de 10 colaboradores previamente selecionados conforme o Programa de Prevenção de Riscos Ambientais da empresa. Cada amostra obtida correspondeu a um tempo superior a $70 \%$ da jornada diária de trabalho de cada colaborador. Os valores das leituras de ruído foram apresentados com média das medições para cada colaborador.

\section{RESULTADOS E DISCUSSÃO}

Com o intuito de regulamentar a exposição dos trabalhadores ao ruído intenso, evitando a surdez ocupacional, as legislações em todo o mundo, adotam critérios estabelecendo formas de intervenções baseadas em ações preventivas das 
alterações auditivas de origem ocupacional. Assim sendo, no Brasil e em muitos outros países, para a regulamentação das condições dos ambientes de trabalho, utiliza-se do modelo do Limite de Tolerância (LT), que corresponde à concentração ou à intensidade mínima ou máxima de agentes de risco a que o trabalhador pode se expor [8]. Neste estudo foram avaliados 10 colaboradores e cada amostra obtida correspondeu a um tempo superior a $70 \%$ da jornada diária de trabalho de cada colaborador. Os dados obtidos nas campanhas de monitoramento encontram-se na Tabela 1. Considerando a série de dados obtidos pode-se observar que os valores das leituras de ruído foram entre 75,6 dB (para o colaborador com menor exposição) a $86,0 \mathrm{~dB}$ (para o colaborador com maior exposição).

Tabela 1 Dados obtidos nas campanhas de monitoramento

\begin{tabular}{|c|c|c|c|}
\hline Cargo Avaliado & $\begin{array}{l}\text { Identificação } \\
\text { da amostra }\end{array}$ & $\begin{array}{c}\text { Nível } \\
\text { Ponderado }\end{array}$ & Parecer Técnico \\
\hline Eletricista & Ammnt-01 & $81,9 \mathrm{~dB}(\mathrm{~A})$ & $\begin{array}{c}\text { Exposição Abaixo do L.T e no Níve } \\
\text { de Ação }\end{array}$ \\
\hline Pedreiro & Ammnt-02 & $80,8 \mathrm{~dB}(\mathrm{~A})$ & $\begin{array}{c}\text { Exposição Abaixo do L.T e no Nível } \\
\text { de Ação }\end{array}$ \\
\hline $\begin{array}{l}\text { Mecânico de } \\
\text { manutenção }\end{array}$ & Ammnt-03 & $81,3 \mathrm{~dB}(\mathrm{~A})$ & $\begin{array}{c}\text { Exposição Abaixo do L.T e no Nível } \\
\text { de Ação }\end{array}$ \\
\hline Marceneiro & Ammnt-04 & $82,3 \mathrm{~dB}(\mathrm{~A})$ & $\begin{array}{c}\text { Exposição Abaixo do L.T e no Níve } \\
\text { de Ação }\end{array}$ \\
\hline Auxiliar de Manutenção & Ammnt-05 & $75,6 \mathrm{~dB}(\mathrm{~A})$ & $\begin{array}{l}\text { Exposição Abaixo do L.T e do Nível } \\
\text { de Ação. }\end{array}$ \\
\hline Serralheiro & Ammnt-06 & $81,9 \mathrm{~dB}(\mathrm{~A})$ & $\begin{array}{c}\text { Exposição Abaixo do L.T e no Nível } \\
\text { de Acão }\end{array}$ \\
\hline Operador de Caldeira & Ammnt-07 & $86,0 \mathrm{~dB}(\mathrm{~A})$ & $\begin{array}{l}\text { Exposição ACIMA do L.T e do Nível } \\
\text { de Ação. }\end{array}$ \\
\hline Pintor & Ammnt-08 & $83,6 \mathrm{~dB}(\mathrm{~A})$ & $\begin{array}{c}\text { Exposição Abaixo do L.T e no Níve } \\
\text { de Ação }\end{array}$ \\
\hline $\begin{array}{l}\text { Supervisor de } \\
\text { Manutenção }\end{array}$ & Ammnt-09 & $78,9 \mathrm{~dB}(\mathrm{~A})$ & $\begin{array}{c}\text { Exposição Abaixo do L.T e no Nível } \\
\text { de Ação }\end{array}$ \\
\hline Chapista & Ammnt-10 & $85,2 \mathrm{~dB}(\mathrm{~A})$ & $\begin{array}{l}\text { Exposição ACIMA do L.T e do Nível } \\
\text { de Ação. }\end{array}$ \\
\hline
\end{tabular}

Obs.: 1 L.T - Limite de Tolerância Nível de Ação= Qualquer valor compreendido entre 80 e 85 dB(A), ou seja, entre $50 \%$ e $100 \%$ da Dose

Observando-se os dados apresentados na Tabela 1 é possível identificar que as médias dos níveis de ruído apresentam-se superiores ao nível de ação recomendado pela NR-15 Anexo-01, que estabelece o padrão de $85 \mathrm{~dB}(\mathrm{~A})$ / 8,0 de exposição diária. Acredita-se que os principais responsáveis pelo elevado nível de ruído provocado pela atividade sejam equipamentos diversos utilizados nos serviços, geradores de energia elétrica, entre outros. Dessa forma, espera-se que os resultados obtidos neste mapeamento preliminar acústico, associado a uma modelagem de parâmetros matemáticos (etapa posterior do estudo) possam auxiliar no planejamento da implantação de uma gestão mais eficaz, gerando um número menor de afastamentos relacionados geração de ruídos, e consequentemente o aumento da produção. Além disso, a identificação prévia dos níveis de ruídos e intervenções necessárias garantem 
o bem-estar e melhor condições de trabalho, afetando de forma positiva a saúde dos trabalhadores investigados.

A perda de audição induzida pelo ruído é causada, normalmente, pela exposição prolongada a níveis de ruído elevados. O primeiro sintoma costuma ser a incapacidade de ouvir sons agudos. Se o problema de excesso de ruído não for solucionado, a audição continuará a deteriorar-se, com perda de capacidade para ouvir sons graves e geralmente o problema afeta os dois ouvidos. Os danos da perda de audição induzida pelo ruído são permanentes, contudo, a perda de audição pode ocorrer sem exposição prolongada. A exposição breve a ruídos impulsivos (ou mesmo a um único impulso forte), como os produzidos pelo disparo de uma arma de fogo, pelo impacto de um martelo ou de um martelo pneumático de rebitar, podem ter efeitos permanentes, incluindo a perda da audição (zumbido) contínuo. Os impulsos podem ainda perfurar a membrana do tímpano, problema que, apesar de doloroso, é reversível [11].

É importante destacar que os níveis de ruído acima dos limites de tolerância preconizados pela legislação atual, indica a necessidade de adoção de medidas para a sua eliminação, através de programas específicos. Quando o ambiente de trabalho apresenta níveis elevados de pressão sonora por exemplo, estes devem ser controlados de modo que o trabalhador possa dar continuidade às suas funções sem prejuízo adicional à sua saúde.

Dada a interferência negativa do ruído nos aspectos pessoais, biológicos e sociais dos trabalhadores, é de suma importância o desenvolvimento de pesquisas que visem identificar e avaliar a forma que a exposição a níveis de pressão sonora elevados pode interferir na qualidade de vida destes trabalhadores. Além disso, face às diversas considerações, mas principalmente da necessidade de melhor orientar a adoção de medidas de proteção aos trabalhadores contra riscos ambientais, a legislação nacional normatiza conceitos, etapas, procedimentos, a serem utilizados em um programa de Higiene do Trabalho, a qual denominou Programa de Prevenção de Riscos Ambientais (PPRA). Este deve apresentar estratégias de ação, divulgação, avaliação e fiscalização quanto ao prazo e o andamento das metas. Essa obrigatoriedade acarreta na criação de parâmetros mínimos estabelecidos nas empresas, o qual se refere a antecipar, reconhecer, avaliar e controlar os riscos.

\section{CONCLUSÃO}

A partir dos resultados obtidos para os 10 colaboradores avaliados no ambiente de manutenção da empresa, identificou-se que as médias dos níveis de ruído apresentam-se superiores ao nível de ação recomendado pela NR-15.

De acordo com a avaliação do risco ambiental efetuado na empresa estudada, e levando em conta a legislação vigente e confrontando os valores por ela preconizados, como limites de tolerância, com os valores resultantes das avaliações, evidencia-se que os resultados e conclusões não devem ser entendidos como definitivos, e que para conhecimento real dos problemas faz-se necessário o desenvolvimento de programas de monitoramento ambiental regular. Entretanto, tendo em vista que os 
resultados obtidos estavam acima dos limites preconizados, medidas de intervenção são necessárias para evitar problemas futuros na saúde dos trabalhadores.

\section{Agradecimentos}

Os autores agradecem a Fundação de Amparo à Pesquisa do Estado da Bahia FAPESB pela bolsa de pesquisa.

\section{REFERÊNCIAS}

1M SEGRE, Marco; FERRAZ, Flávio Carvalho. O conceito de saúde. Rev. Saúde Pública [online]. 1997, vol.31, n.5, pp.538-542.

2GIANNINI, Camila Fernanda et al. AVALIAÇÃO DO RUÍDO AMBIENTAL: MONITORAMENTO E CARACTERIZAÇÃO DO RUÍDO DE FUNDO EM MARINGÁ. Disponível em: <http://www.cesu mar.br/prppge/pesquisa/epcc2011/anais/camila_fernanda_giannini.pdf $>$. Acesso em: 08 mai. 2019.

3VENTUROLI, Fábio; FIEDLER, Nilton C.; MINETTI, Luciano J.; MARTINS, Ildeu S. Avaliação do nível de ruído em marcenarias no Distrito Federal, Brasil. Rev. bras. eng. agríc. ambient. 2003, vol.7, n.3, pp.547-551

${ }^{4}$ ILDA, Itiro. Ergonomia - projeto e produção. 3aㅗ ed, São Paulo Edigard Blücher, $1995 \mathrm{p}$.

${ }^{5}$ BRASIL. Ministério do Trabalho. Portaria n. 3214 de 08 de junho de 1978. Aprova as normas regulamentadoras do Capítulo V,NR-06, Título II, das leis do trabalho, relativas a segurança e medicina do trabalho. Diário Oficial da República Federativa do Brasil, Brasília (DF); 1978 Jul 6.

${ }^{6}$ OLIVA, Flavia Cardoso et al. Mudança significativa do limiar auditivo em trabalhadores expostos a diferentes níveis de ruído. Rev. soc. bras. fonoaudiol. 2011, vol.16, n.3, pp.260-265.

${ }^{7}$ Brasil. Ministério do Trabalho. Portaria n. 3214 de 08 de junho de 1978. Aprova as normas regulamentadoras do Capítulo V, NR-09, Título II, das leis do trabalho, relativas a segurança e medicina do trabalho. Diário Oficial da República Federativa do Brasil, Brasília (DF); 1978 Jul 6.

${ }^{8}$ Girardi, Gustavo, Sellitto. Medição e reconhecimento do risco físico ruído em uma indústria moveleira.

${ }^{9}$ BRASILI. Ministério do Trabalho. Portaria n. 3214 de 08 de junho de 1978. Aprova as normas regulamentadoras do Capítulo V, NR-15, Título II, das leis do trabalho, relativas a segurança e medicina do trabalho. Diário Oficial da República Federativa do Brasil, Brasília (DF); 1978 Jul 6.

${ }^{10}$ BRASIL, Ministério do Trabalho e Emprego. Norma de Higiene Ocupacional NHO 01 - Avaliação da exposição ocupacional ao ruído, FUNDACENTRO, 2001

${ }^{11}$ Agência Europeia para a Segurança e a Saúde no Trabalho, O impacto do ruído 
no trabalho, 2005. 